Pacific Journal of Mathematics

A TWO-POINT BOUNDARY PROBLEM FOR
NONHOMOGENEOUS SECOND ORDER DIFFERENTIAL

TEPHEN TEFTELLE 


\title{
A TWO-POINT BOUNDARY PROBLEM FOR NONHOMOGENEOUS SECOND ORDER DIFFERENTIAL EQUATIONS
}

\author{
S. C. Tefteller
}

This paper is concerned with second order nonhomogeneous differential equations, together with boundary conditions specified at two points. The existence of eigenvalues is established and the oscillatory behavior of the associated eigenfunctions is studied. The results of this paper are obtained by considering the nonhomogeneous problem without regard for existence of solutions of the associated homogeneous boundary problem.

Consider the linear differential equation

$$
\left(r(x, \lambda) y^{\prime}\right)^{\prime}+q(x, \lambda) y=f(x, \lambda),
$$

and the associated homogeneous equation

$$
\left(r(x, \lambda) u^{\prime}\right)^{\prime}+q(x, \lambda) u=0,
$$

where $r(x, \lambda), q(x, \lambda)$, and $f(x, \lambda)$ are real-valued functions on $X: a \leqq$ $x \leqq b, L: \lambda_{\#}-\delta<\lambda<\lambda_{\sharp}+\delta, 0<\delta \leqq \infty,-\infty<a<b<\infty$. We shall consider (1) together with two-point boundary conditions of the form

(a) $\alpha(\lambda) y(a, \lambda)-\beta(\lambda)\left(r y^{\prime}\right)(\alpha, \lambda)=0$,

(b) $\gamma(\lambda) y(b, \lambda)-\delta(\lambda)\left(r y^{\prime}\right)(b, \lambda)=0$.

It is well known that for those values of $\lambda$ for which the associated homogeneous boundary problem $(2,3)$ has no solution, the nonhomogeneous problem (1.3) yields a unique solution. Further, for those values of $\lambda$ for which $(2,3)$ has a solution, the problem $(1,3)$ either has no solution or an infinite number of solutions.

In either case the homogeneous problem must be solved or shown to have only the trivial solution. This paper establishes the existence of characteristic values for $(1,3)$ independent of the corresponding reduced problem. The methods used will be analogous to those of W. M. Whyburn $[6,7,8]$, and G. J. Etgen [2, 3].

The following hypotheses on the coefficients involved in the boundary problem will be assumed throughout:

$\left(\mathrm{H}_{1}\right)$ For each $x \in X$, each of $r(x, \lambda), q(x, \lambda)$, and $f(x, \lambda)$ is continuous on $L$.

$\left(\mathrm{H}_{2}\right)$ For each $\lambda \in L$, each of $r(x, \lambda), q(x, \lambda)$, and $f(x, \lambda)$ is measurable on $X$. 
$\left(\mathrm{H}_{3}\right)$ There exists a Lebesgue integrable function $M(x)$ on $X$ such that $|1 / r(x, \lambda)| \leqq M(x),|q(x, \lambda)| \leqq M(x)$, and $|f(x, \lambda)| \leqq M(x)$ on $X L$.

$\left(\mathrm{H}_{4}\right) \quad r(x, \lambda)>0$ on $X L$.

$\left(\mathrm{H}_{5}\right)$ Each of the functions $\alpha(\lambda), \beta(\lambda), \gamma(\lambda)$, and $\delta(\lambda)$ is continuous on $L$.

$\left(\mathrm{H}_{6}\right) \quad \alpha^{2}(\lambda)+\beta^{2}(\lambda)>0$ and $\gamma^{2}(\lambda)+\delta^{2}(\lambda)>0$ on $L$. In particular, without loss of generality, we assume $\alpha^{2}(\lambda)+\beta^{2}(\lambda) \equiv 1$ on $L$.

$\left(\mathrm{H}_{7}\right) \quad \delta(\lambda)>0$ on $L$. Also, without loss of generality, we assume $0<\arcsin \left(\delta\left(\lambda_{\sharp}\right) /\left[\gamma^{2}\left(\lambda_{\sharp}\right)+\delta^{2}\left(\lambda_{\sharp}\right)\right]^{1 / 2}\right)<\pi$.

2. Preliminary definitions and results. Hypotheses $\mathrm{H}_{1}-\mathrm{H}_{3}$ allow the application of fundamental existence and uniqueness theorems [1, Ch. 2] for differential equations to obtain the existence of a pair of solutions $\{u(x, \lambda), v(x, \lambda)\}$ of (2) such that $W(x, \lambda) \equiv 1$ on $X L$, where $W(x, \lambda)=r(x, \lambda)\left[v^{\prime}(x, \lambda) u(x, \lambda)-u^{\prime}(x, \lambda) v(x, \lambda)\right]$. Such a pair of solutions will be called a normalized solution basis of (2). It is now easily verified that given a normalized solution basis $\{u(x, \lambda), v(x, \lambda)\}$ of (2), every solution of (1) is of the form

$$
\begin{aligned}
y(x, \lambda)= & {\left[c_{1}(\lambda)-\int_{a}^{x} f(t, \lambda) v(t, \lambda) d t\right] u(x, \lambda) } \\
& +\left[c_{2}(\lambda)+\int_{a}^{x} f(t, \lambda) u(t, \lambda) d t\right] v(x, \lambda) .
\end{aligned}
$$

Moreover, there exists a solution $y(x, \lambda)$ of (1) satisfying

$$
y(a, \lambda) \equiv \beta(\lambda),\left(r y^{\prime}\right)(\alpha, \lambda) \equiv \alpha(\lambda)
$$

on $L$. In fact, if $\{u(x, \lambda), v(x, \lambda)\}$ is the normalized solution basis of (2) satisfying the initial conditions

$$
\begin{aligned}
u(a, \lambda) & \equiv 1, v(a, \lambda) \equiv 0, \\
\left(r u^{\prime}\right)(a, \lambda) & \equiv 0,\left(r v^{\prime}\right)(a, \lambda) \equiv 1,
\end{aligned}
$$

on $L$, then

$$
\begin{aligned}
y(x, \lambda)= & {\left[\beta(\lambda)-\int_{a}^{x} f(t, \lambda) v(t, \lambda) d t\right] u(x, \lambda) } \\
& +\left[\alpha(\lambda)+\int_{a}^{x} f(t, \lambda) u(t, \lambda) d t\right] v(x, \lambda)
\end{aligned}
$$

satisfies (5). Thus the solution $y(x, \lambda)$ defined by (7) satisfies (3a).

We establish the existence of values of $\lambda$ on $L$ for which there corresponds a solution of (1) satisfying (3a, b). Such values are called eigenvalues of the respective boundary problem.

Let $\{u(x, \lambda), v(x, \lambda)\}$ be the normalized solution basis of (2) defined by (6). Applying the polar coordinate transformation, we obtain 


$$
\begin{aligned}
u(x, \lambda) & =\rho_{1}(x, \lambda) \sin \theta_{1}(x, \lambda), v(x, \lambda)=\rho_{2}(x, \lambda) \sin \theta_{2}(x, \lambda), \\
\left(r u^{\prime}\right)(x, \lambda) & =\rho_{1}(x, \lambda) \cos \theta_{1}(x, \lambda),\left(r v^{\prime}\right)(x, \lambda)=\rho_{2}(x, \lambda) \cos \theta_{2}(x, \lambda),
\end{aligned}
$$

where $\rho_{i}(x, \lambda)$ and $\theta_{i}(x, \lambda)$ are solutions of

$$
\begin{aligned}
& \rho_{i}^{\prime}(x, \lambda)=\rho_{i}(x, \lambda)\left[\frac{1}{r(x, \lambda)}-q(x, \lambda)\right] \sin \theta_{i}(x, \lambda) \cos \theta_{i}(x, \lambda) \\
& \theta_{i}^{\prime}(x, \lambda)=\frac{1}{r(x, \lambda)} \cos ^{2} \theta_{i}(x, \lambda)+q(x, \lambda) \sin ^{2} \theta_{i}(x, \lambda)
\end{aligned}
$$

$i=1,2$, satisfying $\rho_{1}(a, \lambda) \equiv \rho_{2}(a, \lambda) \equiv 1, \theta_{1}(a, \lambda) \equiv \pi / 2, \theta_{2}(a, \lambda) \equiv 0$ on $L$.

LEMma 1. The following inequality holds on $X L: 0<\theta_{1}(x, \lambda)-$ $\theta_{2}(x, \lambda)<\pi$.

Proof. Using the polar form of $u(x, \lambda)$ and $v(x, \lambda)$, it follows that

$$
1 \equiv W(x, \lambda)=\rho_{1}(x, \lambda) \rho_{2}(x, \lambda) \sin \left[\theta_{1}(x, \lambda)-\theta_{2}(x, \lambda)\right],
$$

where $W(x, \lambda)=r(x, \lambda)\left[v^{\prime} u-u^{\prime} v\right]$. Hence $\sin \left[\theta_{1}(x, \lambda)-\theta_{2}(x, \lambda)\right]=$ $1 / \rho_{1}(x, \lambda) \rho_{2}(x, \lambda)>0$ on $X L$. Since $\theta_{1}(a, \lambda)-\theta_{2}(a, \lambda) \equiv \pi / 2$ on $L$, we have $0<\theta_{1}(x, \lambda)-\theta_{2}(x, \lambda)<\pi$ on $X L$.

CoRollary. For each $x \in X$, the zeros of $u(x, \lambda)$ and $v(x, \lambda)$ separate each other on $L$.

We can write (7) as $y(x, \lambda)=A(x, \lambda) u(x, \lambda)+B(x, \lambda) v(x, \lambda)$, where

$$
\begin{aligned}
& A(x, \lambda)=\beta(\lambda)-\int_{a}^{x} f(t, \lambda) v(t, \lambda) d t, \quad \text { and } \\
& B(x, \lambda)=\alpha(\lambda)+\int_{a}^{x} f(t, \lambda) u(t, \lambda) d t .
\end{aligned}
$$

It then follows that $y(\bar{x}, \lambda)=y^{\prime}(\bar{x}, \lambda)=0$ for some $\bar{x} \in X$ if and only if $A(\bar{x}, \lambda)=B(\bar{x}, \lambda)=0$, [Lemma 3.3, Theorem 3.12; 5].

If for some $\lambda=\bar{\lambda}, y(b, \bar{\lambda})=y^{\prime}(b, \bar{\lambda})=0$, where the solution $y(x, \lambda)$ is defined by (7), then the boundary condition (3b) is satisfied and $\bar{\lambda}$ is an eigenvalue. We note this possibility could be ruled out if we assume that $\beta(\lambda)+1>\exp \int_{a}^{b} M(t) d t$ on $L$, where $M(t)$ is defined in $\mathrm{H}_{3}$ [Theorem $3.4,5]$. So in the following we assume $y(b, \lambda)$ has no double zeros on $L$.

In order to establish the existence of eigenvalues for $(1,3 \mathrm{a}, \mathrm{b})$, we introduce the functions

$$
\begin{aligned}
& U(x, \lambda)=\gamma(\lambda) u(x, \lambda)-\delta(\lambda)\left(r u^{\prime}\right)(x, \lambda), \\
& V(x, \lambda)=\gamma(\lambda) v(x, \lambda)-\delta(\lambda)\left(r v^{\prime}\right)(x, \lambda),
\end{aligned}
$$


and

$$
\begin{aligned}
& s(x, \lambda)=A(b, \lambda) U(x, \lambda)+B(b, \lambda) V(x, \lambda), \\
& t(x, \lambda)=A(b, \lambda) V(x, \lambda)-B(b, \lambda) U(x, \lambda),
\end{aligned}
$$

where $\{u(x, \lambda), v(x, \lambda)\}$ is the solution basis of (2) defined by (6), and where $A(b, \lambda)$ and $B(b, \lambda)$ are defined by $(9)$.

It follows that

$$
s^{2}(x, \lambda)+t^{2}(x, \lambda)=\left[A^{2}(b, \lambda)+B^{2}(b, \lambda)\right]\left[U^{2}(x, \lambda)+V^{2}(x, \lambda)\right] .
$$

Writing $u(x, \lambda)$ and $v(x, \lambda)$ in polar form, we have

$$
\begin{aligned}
& s^{2}(x, \lambda)+t^{2}(x, \lambda)=\left(A^{2}(b, \lambda)+B^{2}(b, \lambda)\right)\left(\gamma^{2}(\lambda)+\delta^{2}(\lambda)\right) . \\
& \quad\left(\rho_{1}^{2}(x, \lambda) \sin ^{2}\left[\theta_{1}(x, \lambda)-\tau(\lambda)\right]+\rho_{2}^{2}(x, \lambda) \sin ^{2}\left[\theta_{2}(x, \lambda)-\tau(\lambda)\right]\right),
\end{aligned}
$$

where

$$
\begin{aligned}
& \sin \tau(\lambda)=\delta(\lambda) /\left[\gamma^{2}(\lambda)+\delta^{2}(\lambda)\right]^{1 / 2}, \quad \text { and } \\
& \cos \tau(\lambda)=\gamma(\lambda) /\left[\gamma^{2}(\lambda)+\delta^{2}(\lambda)\right]^{1 / 2} .
\end{aligned}
$$

From Lemma 1, we have that $0<\left[\theta_{1}(x, \lambda)-\tau(\lambda)\right]-\left[\theta_{2}(x, \lambda)-\tau(\lambda)\right]<$ $\pi$ on $X L$, implying $\sin \left[\theta_{1}(x, \lambda)-\tau(\lambda)\right]$ and $\sin \left[\theta_{2}(x, \lambda)-\tau(\lambda)\right]$ cannot vanish simultaneously for any $x \in X, \lambda \in L$. Using $\mathrm{H}_{6}$, we conclude that $U^{2}(x, \lambda)+V^{2}(x, \lambda)>0$ on $X L$. By our assumption that $y(b, \lambda)$ has no double zeros on $L, A^{2}(b, \lambda)+B^{2}(b, \lambda)>0$ on $L$. Consequently, $s^{2}(x, \lambda)+t^{2}(x, \lambda)>0$ on $X L$ and the complex-valued function $\Delta(x, \lambda)$, defined by

$$
\Delta(x, \lambda)=(s(x, \lambda)+i t(x, \lambda)) /(s(x, \lambda)-i t(x, \lambda))
$$

exists on $X L$.

THEOREM 1. The complex-valued function $\Delta(x, \lambda)$ has the following properties on $X$ for each $\lambda \in L$ :

(i ) $|\Delta(x, \lambda)|=1$.

(ii) $\Delta(x, \lambda)$ satisfies the first order equation $d \Delta(x, \lambda) / d x=$ $2 i \Delta(x, \lambda) h(x, \lambda)$, where

$$
h(x, \lambda)=\left(s(x, \lambda) t^{\prime}(x, \lambda)-s^{\prime}(x, \lambda) t(x, \lambda)\right) /\left(s^{2}(x, \lambda)+t^{2}(x, \lambda)\right) .
$$

(iii) $\Delta(x, \lambda)=1$ if and only if $t(x, \lambda)=0$,

$$
\Delta(x, \lambda)=-1 \text { if and only if } s(x, \lambda)=0 \text {. }
$$

(iv) Let $\sigma(x, \lambda)=\arg \Delta(x, \lambda)$, where it is assumed that $0 \leqq$ $\sigma\left(\alpha, \lambda_{\sharp}\right)<2 \pi$ and that $\sigma(x, \lambda)$ is continued as a continuous function on $X L$. Then, for each fixed $\lambda$,

$$
2 \int_{a}^{x} h(w, \lambda) d w=\sigma(x, \lambda)-\sigma(a, \lambda) .
$$


( v ) If $\gamma^{2}(\lambda) / r(x, \lambda)+\delta^{2}(\lambda) q(x, \lambda)>0$ on $X L$, then $\Delta(x, \lambda)$ moves monotonically and positively on the unit circle.

Proof. Properties (i)-(iii) are easily verified. Equation (15) is a result of solving the first order equation in $\Delta(x, \lambda)$ and applying the definition of $\sigma(x, \lambda)$. To prove $(\mathrm{v})$, we note that $h(x, \lambda)=\left(V U^{\prime}-\right.$ $\left.U V^{\prime}\right) /\left(U^{2}+V^{2}\right)=\left(\gamma^{2}(\lambda) / r(x, \lambda)+\delta^{2}(\lambda) q(x, \lambda)\right)\left(u r v^{\prime}-v r u^{\prime}\right) /\left(U^{2}+V^{2}\right)$. Since $u r v^{\prime}-v r u^{\prime} \equiv 1, h(x, \lambda)>0$ on $X L$, and $\sigma(x, \lambda)$ is monotone increasing if $\gamma^{2}(\lambda) / r(x, \lambda)+\delta^{2}(\lambda) q(x, \lambda)$ is positive on $X L$.

Considering equation (13), we note that $\Delta(a, \lambda)=(B(b, \lambda)-i A(b$, $\lambda)) /(B(b, \lambda)+i A(b, \lambda)) \neq 1$ on $L$. Thus $0<\sigma(a, \lambda)<2 \pi$ on $L$, and

$$
2 \int_{a}^{b} h(w, \lambda) d w<\sigma(b, \lambda)<2 \int_{a}^{b} h(w, \lambda) d w+2 \pi
$$

on $L$.

3. Existence of eigenvalues. Using the results of the preceding section, we can now state an existence theorem for eigenvalues of $(1,3 a, b)$.

THEOREM 2. Let $y(x, \lambda)$ be the solution of $(1,3 \mathrm{a})$, where $y(x, \lambda)$ is defined by (7). Define $Q(\lambda)$ by

$$
Q(\lambda)=2 \int_{a}^{b} h(w, \lambda) d w
$$

$\left(h(w, \lambda)\right.$ defined by (14)). Suppose $\gamma^{2}(\lambda) / r(x, \lambda)+\delta^{2}(\lambda) q(x, \lambda)>0$ on $X L$. Then $Q(\lambda)>0$ on $L$. Let $m \geqq 0$ be the least integer such that inf $Q(\lambda)<(2 m+1) \pi$ on $L$, and let $n$ be an integer such that sup $Q(\lambda)>(2 n+1) \pi$ on $L$. If $n \geqq m+1$, then there exist $p, p=n-m$, eigenvalues $\lambda_{0}, \lambda_{1}, \cdots, \lambda_{p_{-1}}$ of $(1,3 \mathrm{a}, \mathrm{b})$.

Proof. Let $y(x, \lambda)$ be the solution of (1) defined by (7). Let $U(x, \lambda)$, $V(x, \lambda), s(x, \lambda), t(x, \lambda)$, and $\Delta(x, \lambda)$ be defined as above.

If $\gamma^{2}(\lambda) / r(x, \lambda)+\delta^{2}(\lambda) q(x, \lambda)>0$ on $X L$, then from Theorem 1 , we know $\sigma(b, \lambda)-\sigma(a, \lambda)>0$ on $L$, and $Q(\lambda)>0$ on $L$.

Suppose that $m$ and $n$ are integers with the properties described in the hypothesis. Then there exists a value of $\lambda$, say $\lambda^{*}$, such that $Q\left(\lambda^{*}\right)<(2 m+1) \pi$ and a value of $\lambda$, say $\bar{\lambda}$, such that $Q(\bar{\lambda})>(2 n+$ 1) $\pi$. Clearly, $\lambda^{*} \neq \bar{\lambda}$, and so we may assume $\lambda^{*}<\bar{\lambda}$. From (16), we have $Q(\lambda)<\sigma(b, \lambda)<Q(\lambda)+2 \pi$ on $L$. Therefore, $\sigma\left(b, \lambda^{*}\right)<(2 m+$ $3) \pi$ and $\sigma(b, \bar{\lambda})>(2 n+1) \pi$. Since $n=m+p, p \geqq 1$, there exist $p$ values of $\lambda, \lambda_{0}, \lambda_{1}, \cdots, \lambda_{p_{-1}}$ on $\left(\lambda^{*}, \bar{\lambda}\right)$ such that $\sigma\left(b, \lambda_{j}\right)=[2(m+j)+$ $3] \pi$, for $j=0,1, \cdots, p-1$. We assume that $\lambda_{0}<\lambda_{1}<\cdots<\lambda_{p-1}$ since $\sigma(b, \lambda)$ is continuous in $\lambda$. Now $\sigma(b, \lambda)=\arg \Delta(x, \lambda)$ implies that $\Delta\left(b, \lambda_{j}\right)=-1$ for $j=0,1, \cdots, p-1$, and consequently, $s\left(b, \lambda_{j}\right)=0$ 
for $j=0,1, \cdots, p-1$.

Considering (3b) we have

$$
\begin{aligned}
\gamma(\lambda) y(b, \lambda)-\delta(\lambda)\left(r y^{\prime}\right)(b, \lambda) & =A(b, \lambda) U(b, \lambda)+B(b, \lambda) V(b, \lambda) \\
& =s(b, \lambda) .
\end{aligned}
$$

Hence for $\lambda_{j}, j=0,1, \cdots, p-1$, the condition $(3 b)$ is satisfied and the $\lambda_{j}$ are the eigenvalues for $(1,3 a, b)$.

CoROLLARY. Under the hypotheses of Theorem 2, if the integer $n$ can be chosen arbitrary large, then there exist infinitely many eigenvalues $\lambda_{0}, \lambda_{1}, \lambda_{2}, \cdots$ for $(1,3 \mathrm{a}, \mathrm{b})$.

The following theorem also gives a criterion for the existence of eigenvalues for this nonhomogeneous boundary problem.

TheOREm 3. Let $y(x, \lambda)$ be the solution of $(1,3 \mathrm{a})$ defined by $(7)$. Then $\theta_{2}(b, \lambda)-\tau(\lambda)>-\pi$ on $L$, where $\theta_{2}(x, \lambda)$ and $\tau(\lambda)$ are defined by (8) and (12) respectively. Suppose $A(b, \lambda) \neq 0$ on $L$, where $A(x, \lambda)$ is defined by (9). Let $m \geqq 0$ be the least integer so that inf $\left[\theta_{2}(b, \lambda)-\right.$ $\tau(\lambda)]<m \pi$ on $L$, and let $n$ be an integer such that $\sup \left[\theta_{2}(b, \lambda)-\right.$ $\tau(\lambda)]>n \pi$ on $L$. If $n \geqq m+2$, then there exist at least $p-1, p=$ $n-m$, nonempty sets of eigenvalues $T_{0}, T_{1}, \cdots, T_{p_{-2}}$ for the boundary problem $(1,3 \mathrm{a}, \mathrm{b})$.

Proof. From (7), (9) and the polar representation for the normalized solution basis $\{u(x, \lambda), v(x, \lambda)\}$ of $(2)$, defined by (6), we have $y(x, \lambda)=$ $A(x, \lambda) \rho_{1}(x, \lambda) \sin \theta_{1}(x, \lambda)+B(x, \lambda) \rho_{2}(x, \lambda) \sin \theta_{2}(x, \lambda)$. Further, we can write the boundary condition (3b) in the form

$$
\begin{aligned}
P(\lambda)= & {\left[\gamma^{2}(\lambda)+\delta^{2}(\lambda)\right]^{1 / 2}\left\{\rho_{1}(b, \lambda) A(b, \lambda) \sin \left[\theta_{1}(b, \lambda)-\tau(\lambda)\right]\right.} \\
& \left.+\rho_{2}(b, \lambda) B(b, \lambda) \sin \left[\theta_{2}(b, \lambda)-\tau(\lambda)\right]\right\},
\end{aligned}
$$

where $\rho_{i}(x, \lambda), \theta_{i}(x, \lambda), i=1,2$ are defined by $(8)$, and $\tau(\lambda)$ is defined by (12).

Since $\theta_{2}^{\prime}(x, \lambda)=1 / r(x, \lambda)>0$ when $v(x, \lambda)=0, \theta_{2}(x, \lambda)$ is increasing at zeros of $v(x, \lambda)$, for each $\lambda \in L$. Moreover, $\theta_{2}(a, \lambda) \equiv 0$ implies $\theta_{2}(b, \lambda)>0$ on $L$. Using (12) and $\mathrm{H}_{7}$, we have $0<\tau(\lambda)<\pi$ on $L$, and thus $\theta_{2}(b, \lambda)-\tau(\lambda)>-\pi$ on $L$.

Let $m$ and $n$ be integers with the properties described in the hypotheses. Then there exist values of $\lambda$, say $\lambda^{*}$ and $\bar{\lambda}$, such that $\theta_{2}\left(b, \lambda^{*}\right)-\tau\left(\lambda^{*}\right)<m \pi$ and $\theta_{2}(b, \bar{\lambda})-\tau(\bar{\lambda})>n \pi$. Clearly, $\lambda^{*} \neq \bar{\lambda}$, so assume $\lambda^{*}<\bar{\lambda}$. Since $n=m+p, p \geqq 2$, there exist $p$ values of $\lambda$, $\lambda_{0}, \lambda_{1}, \cdots, \lambda_{p-1}$, on $\left(\lambda^{*}, \bar{\lambda}\right)$ such that $\theta_{2}\left(b, \lambda_{j}\right)-\tau\left(\lambda_{j}\right)=(m+j) \pi, j=$ $0,1, \cdots, p-1$. From the continuity of $\theta_{2}(b, \lambda)-\tau(\lambda)$ on $L$, we may assume $\lambda_{0}<\lambda_{1}<\cdots<\lambda_{p_{-1}}$. 
By Lemma $1,0<\theta_{1}(b, \lambda)-\theta_{2}(b, \lambda)<\pi$ on $L$, and therefore $0<\left[\theta_{1}(b, \lambda)-\tau(\lambda)\right]-\left[\theta_{2}(b, \lambda)-\tau(\lambda)\right]<\pi$ on $L$, and the zeros of $\sin \left[\theta_{1}(b, \lambda)-\tau(\lambda)\right]$ and $\sin \left[\theta_{2}(b, \lambda)-\tau(\lambda)\right]$ separate each other on $L$. Thus $\sin \left[\theta_{1}\left(b, \lambda_{j}\right)-\tau\left(\lambda_{j}\right)\right]>0$ and $\left.\sin \left[\theta_{1}\left(b, \lambda_{j+1}\right)-\tau\left(\lambda_{j+1}\right)\right)\right]<0$ for $j=0,1, \cdots, p-2$, or vice versa.

Without loss of generality, assume that $A(b, \lambda)>0$ on $L$. Then from (17), $P\left(\lambda_{j}\right)>0$ and $P\left(\lambda_{j+1}\right)<0$ for each $j$, or vice versa. In either case, since $P(\lambda)$ is continuous on $L$, there is a $\bar{\lambda}_{j} \in\left(\lambda_{j}, \lambda_{j+1}\right)$ such that $P\left(\bar{\lambda}_{j}\right)=0$, and $\bar{\lambda}_{j}$ is an eigenvalue for $(1,3 \mathrm{a}, \mathrm{b}), j=0,1,2, p-2$. Letting $T_{j}$ be the set of all eigenvalues on $\left(\lambda_{j}, \lambda_{j+1}\right), j=0,1, \cdots, p-$ 2 the theorem is proved.

COROLLARY 1. Under the hypotheses of Theorem 3, if the integer $n$ can be chosen arbitrarily large, then there exist infinitely many sets of eigenvalues $T_{0}, T_{1}, \cdots$ for $(1,3 a, b)$.

CoRollary 2. Suppose, in addition to the hypotheses of Theorem 3 , that $A(x, \lambda) \neq 0$ on $X$ for each $\lambda \in L$. Then there exist $p-1$ nonempty sets of eigenvalues $J_{0}, J_{1}, \cdots, J_{p_{-2}}$ for $(1,3 \mathrm{a}, \mathrm{b})$ such that if $\rho_{j} \in J_{j}, j=0,1, \cdots, p-2$, then $\theta_{2}\left(b, \rho_{j}\right)-\tau\left(\rho_{j}\right) \geqq(m+j) \pi$. Moreover, if $j \geqq 1$, then the corresponding solution $y\left(x, \rho_{j}\right)$ has at least $j-1$ zeros on $X$.

Proof. We know that $\theta_{2}(b, \lambda)-\tau(\lambda)$ is continuous on $L$ and increases from less than $m \pi$ to more than $n \pi$. Choose $\lambda_{j}$ such that $\theta_{2}(b, \lambda)-\tau(\lambda) \geqq(m+j) \pi$ for $\lambda>\lambda_{j}$, and let $J_{j}$ be the set of eigenvalues on $\left(\lambda_{j}, \lambda_{j+1}\right)$. From Theorem 3, each $J_{j}$ is nonempty.

If for fixed $\lambda, \theta_{2}(b, \lambda)-\theta_{2}(a, \lambda) \geqq q \pi$, then $v(x, \lambda) \equiv 0(\bmod \pi)$ at least $q$ times on $X$. Further, if $A(x, \lambda) \neq 0$ on $X$ for each $\lambda \in L$, then by a generalization of a theorem by Leighton [Thm. 2.1,4], we know that the zeros of $y(x, \lambda)$ and $v(x, \lambda)$ separate on $X$. Suppose $\rho_{j} \in J_{j}, j \geqq 1$. Then $\theta_{2}\left(b, \rho_{j}\right)-\tau\left(\lambda_{j}\right) \geqq(m+j) \pi$. Since $\theta_{2}(a, \lambda) \equiv 0$ on $L$ and $\tau(\lambda)>0$ on $L$, this implies that $\theta_{2}\left(b, \lambda_{j}\right)-\theta_{2}\left(a, \lambda_{j}\right) \geqq(m+j) \pi+$ $\tau\left(\rho_{j}\right) \geqq(m+j) \pi \geqq j \pi$. We conclude that $v\left(x, \rho_{j}\right)$ has at least $j$ zeros on $X$, and consequently, $y\left(x, \rho_{j}\right)$ has at least $j-1$ zeros on $X$.

CoRollary 3. Suppose, in the hypotheses of Theorem 3, we assume that $A(b, \lambda)$ does not change sign on $L$, rather than be nonzero. Then the number of distinct eigenvalues for $(1,3 \mathrm{a}, \mathrm{b})$ is at least $(p-1) / 2$ if $p$ is odd and at least $p / 2$ if $p$ is even.

Proof. Paraphrasing Theorem 3, choose $\lambda_{0}, \lambda_{1}, \cdots, \lambda_{p_{-1}}$ such that $\theta_{2}\left(b, \lambda_{j}\right)-\tau\left(\lambda_{j}\right)=(m+j) \pi$. Then $\sin \left[\theta_{1}\left(b, \lambda_{j}\right)-\tau\left(\lambda_{j}\right)\right]>0$ and $\sin \left[\theta_{1}\left(b, \lambda_{i+1}\right)-\tau\left(\lambda_{j+1}\right)\right]<0$ for $j=0,1, \cdots, p-2$, or vice versa.

Assuming, without loss of generality, that $A(b, \lambda) \geqq 0$ on $L$, we 
have $P\left(\lambda_{j}\right) \geqq 0$ and $P\left(\lambda_{j+1}\right) \leqq 0, j=0,1, \cdots, p-2$, or vice versa. In either case, there is a $\bar{\lambda}_{j} \in\left[\lambda_{j}, \lambda_{j+1}\right]$ such that $P\left(\bar{\lambda}_{j}\right)=0$, and $\bar{\lambda}_{j}$ is an eigenvalue for $(1,3 \mathrm{a}, \mathrm{b})$. Let $T_{j}$ be the set of eigenvalues on $\left[\lambda_{j}, \lambda_{j+1}\right]$, $j=0,1, \cdots, p-2$. Now it may happen that two sets $T_{j}$ and $T_{j+1}$ each contain only one eigenvalue, and moreover, that eigenvalue is a common eigenvalue, namely $\lambda_{j+1}$. We find, therefore, that the number of distinct eigenvalues for $(1,3 \mathrm{a}, \mathrm{b})$ is at least $(p-1) / 2$ if $p$ is odd, and at least $p / 2$ if $p$ is even.

We remark that the hypotheses of Theorem 3 require that $A(b, \lambda)=\beta(\lambda)-\int_{a}^{b} f(t, \lambda) v(t, \lambda) d t \neq 0$ on $L$. This can be verified if we assume

(i) $\beta(\lambda)+1>\exp \int_{a}^{b} M(t) d t$ on $L$,

[Thm. 3.4, 5], or

(ii) (a) $q(x, \lambda)$ is not identically zero on any subinterval of $X$ for each $\lambda \in L$, and is not identically zero on any subinterval of $L$ for each $x \in X$.

(b) $f(x, \lambda) / q(x, \lambda)$ is defined, integrable, nonpositive, and nondecreasing on $X$ for each $\lambda \in L$.

(c) $\rho_{1}(b, \lambda) \geqq \rho_{1}(x, \lambda)$ on $X$ for each $\lambda \in L$.

(d) $\beta(\lambda)>-2 \exp \left[\int_{a}^{b} M(t) d t\right] f(b, \lambda) / q(b, \lambda)$ on $L$,

[Thm. 3.5, 5].

Here $M(t)$ is the Lebesgue integrable bound of the functions $1 / r(x, \lambda)$, $q(x, \lambda)$, and $f(x, \lambda)$.

\section{REFERENCES}

1. E. A. Coddington and N. Levinson, Theory of Ordinary Differential Equations, McGraw-Hill, New York, 1955.

2. G. J. Etgen and S. C. Tefteller, A two-point boundary problem for nonlinear second order differential systems, SIAM J. Math. Anal., 2 (1971), 64-71.

3. - Second order differential equations with general boundary conditions, SIAM J. Math. Anal., 3 (1972), 512-520.

4. W. Leighton, On self-adjoint differential equations of second order, J. London Math. Soc., 27 (1952), 37-47.

5. S. C. Tefteller, Oscillation of Second Order Nonhomogeneous Differential Equations, Ph. D. Dissertation, University of Houston, Houston, Texas, 1972.

6. W. M. Whyburn, Existence and oscillation theorems for non-linear systems of the second order, Trans. Amer. Math. Soc., 30 (1928), 848-854.

7. - A note on a nonself-adjoint differential system of the second order, J. Elisha Mitchell Sci. Soc., 69 (1953), 116-118.

8. - A non-linear boundary value problem for second order differential systems, Pacific J. Math., 5 (1955), 147-160.

Received June 21, 1973. 


\section{PACIFIC JOURNAL OF MATHEMATICS}

\section{EDITORS}

RICHARD ARENS (Managing Editor)

University of California

Los Angeles, California 90024

\section{J. DUGUNDJI}

Department of Mathematics University of Southern California Los Angeles, California 90007

D. Gilbarg and J. Milgram

Stanford University

Stanford, California 94305

University of Washington
Seattle, Washington 98105

ASSOCIATE EDITORS
E. F, BECKENBACH
B. H. NEUMANN
F. WOLF
K. Yoshida

\section{SUPPORTING INSTITUTIONS}

\author{
UNIVERSITY OF BRITISH COLUMBIA \\ CALIFORNIA INSTITUTE OF TECHNOLOGY \\ UNIVERSITY OF CALIFORNIA \\ MONTANA STATE UNIVERSITY \\ UNIVERSITY OF NEVADA \\ NEW MEXICO STATE UNIVERSITY \\ OREGON STATE UNIVERSITY \\ UNIVERSITY OF OREGON \\ OSAKA UNIVERSITY
}

\author{
UNIVERSITY OF SOUTHERN CALIFORNIA \\ STANFORD UNIVERSITY \\ UNIVERSITY OF TOKYO \\ UNIVERSITY OF UTAH \\ WASHINGTON STATE UNIVERSITY \\ UNIVERSITY OF WASHINGTON \\ * * * * \\ AMERICAN MATHEMATICAL SOCIETY \\ NAVAL WEAPONS CENTER
}

The Supporting Institutions listed above contribute to the cost of publication of this Journal, but they are not owners or publishers and have no responsibility for its content or policies.

Mathematical papers intended for publication in the Pacific Journal of Mathematics should be in typed form or offset-reproduced, (not dittoed), double spaced with large margins. Underline Greek letters in red, German in green, and script in blue. The first paragraph or two must be capable of being used separately as a synopsis of the entire paper. Items of the bibliography should not be cited there unless absolutely necessary, in which case they must be identified by author and Journal, rather than by item number. Manuscripts, in duplicate if possible, may be sent to any one of the four editors. Please classify according to the scheme of Math. Rev. Index to Vol. 39. All other communications to the editors should be addressed to the managing editor, or Elaine Barth, University of California, Los Angeles, California, 90024.

100 reprints are provided free for each article, only if page charges have been substantially paid. Additional copies may be obtained at cost in multiples of 50 .

The Pacific of Journal Mathematics is issued monthly as of January 1966. Regular subscription rate: $\$ 72.00$ a year (6 Vols., 12 issues). Special rate: $\$ 36.00$ a year to individual members of supporting institutions.

Subscriptions, orders for back numbers, and changes of address should be sent to Pacific Journal of Mathematics, 103 Highland Boulevard, Berkeley, California, 94708.

PUBLISHED BY PACIFIC JOURNAL OF MATHEMATICS, A NON-PROFIT CORPORATION

Printed at Kokusai Bunken Insatsusha (International Academic Printing Co., Ltd.), 270, 3-chome Totsuka-cho, Shinjuku-ku, Tokyo 160, Japan.

Copyright (C) 1973 by Pacific Journal of Mathematics Manufactured and first issued in Japan 


\section{Pacific Journal of Mathematics}

\section{Vol. 53, No. $2 \quad$ April, 1974}

Kenneth Abernethy, On characterizing certain classses of first countable spaces by

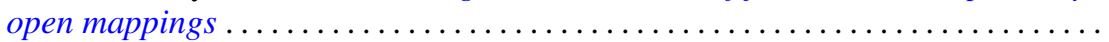

Ross A. Beaumont and Donald Lawver, Strongly semisimple abelian groups .......

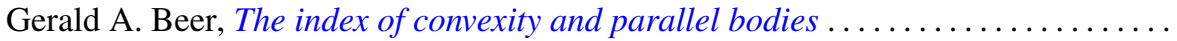

Victor P. Camillo and Kent Ralph Fuller, On Loewy length of rings ..............

Stephen LaVern Campbell, Linear operators for which $T^{*} T$ and $T T^{*}$ commute.

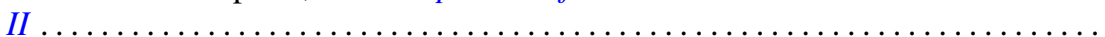

Charles Kam-Tai Chui and Philip Wesley Smith, Characterization of a function by

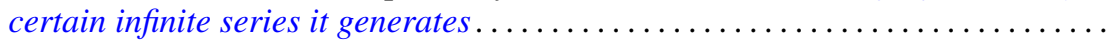

Allan L. Edelson, Conjugations on stably almost complex manifolds . ...........

Patrick John Fleury, Hollow modules and local endomorphism rings . . ..........

Jack Tilden Goodykoontz, Jr., Connectedness im kleinen and local connectedness in

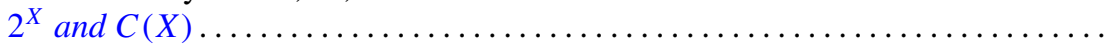

Robert Edward Jamison, II, Functional representation of algebraic intervals .......

Athanassios G. Kartsatos, Nonzero solutions to boundary value problems for

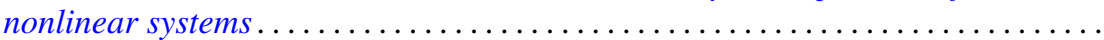

Soon-Kyu Kim, Dennis McGavran and Jingyal Pak, Torus group actions on simply

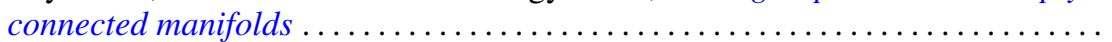

David Anthony Klarner and R. Rado, Arithmetic properties of certain recursively

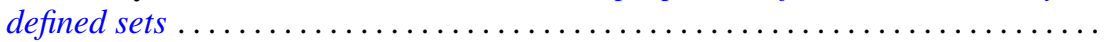

Ray Alden Kunze, On the Frobenius reciprocity theorem for square-integrable

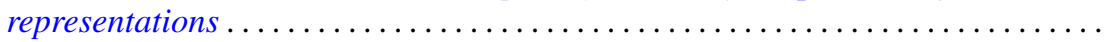

John Lagnese, Existence, uniqueness and limiting behavior of solutions of a class of differential equations in Banach space...

Teck Cheong Lim, A fixed point theorem for families on nonexpansive mappings Lewis Lum, A quasi order characterization of smooth continua

Andy R. Magid, Principal homogeneous spaces and Galois extensions . .

Charles Alan McCarthy, The norm of a certain derivation ..... . .

Louise Elizabeth Moser, On the impossibility of obtaining $S^{2} \times S^{1}$ by elementary surgery along a knot. .

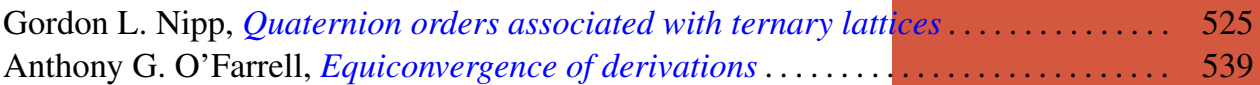

Dorte Olesen, Derivations of $A W^{*}$-algebras are inner . . . . . . . . . . . . . . . 555

Dorte Olesen and Gert Kjærgaard Pedersen, Derivations of $C^{*}$-algebras have

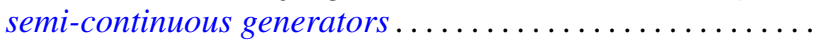

Duane O’Neill, On conjugation cobordism.

Chull Park and S. R. Paranjape, Probabilities of Wiener paths crossing differentiable

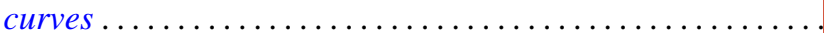

Edward Ralph Rozema, Almost Chebyshev subspaces of $L^{1}(\mu$;

Lesley Millman Sibner and Robert Jules Sibner, A note on the Atiyah-Bott fixed

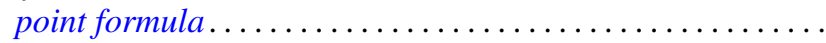

Betty Salzberg Stark, Irreducible subgroups of orthogonal groups generated by

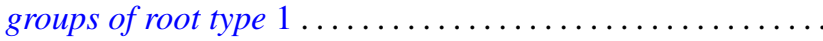

N. Stavrakas, A note on starshaped sets, $(k)$-extreme points and the half ray

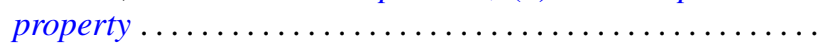

Carl E. Swenson, Direct sum subset decompositions of $Z \ldots \ldots$ 